\title{
Rapidly and Slowly Growing Lineages in Chromosomal Instability-Type Gland-Forming Gastric Carcinomas as Revealed by Multisampling Analysis of DNA Copy-Number Profile
}

\author{
Tu Thanh Duong a, biem Thi-Ngoc Vo ${ }^{b}$ Takahisa Nakayama ${ }^{a}$ \\ Ken-ichi Mukaisho ${ }^{a}$ Masamichi Bamba ${ }^{c}$ Trung Sao Nguyen ${ }^{b}$ \\ Hiroyuki Sugihara ${ }^{a}$

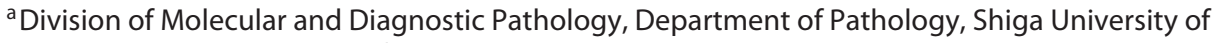 \\ Medical Science, Ōtsu, Japan; ${ }^{b}$ Department of Pathology, Ho Chi Minh City University of Medicine and \\ Pharmacy, Ho Chi Minh City, Vietnam; ' Department of Pathology, Saiseikai Shiga Hospital, Imperial \\ Gift Foundation Inc., Rittō, Japan
}

\author{
Keywords \\ Stomach - Adenocarcinoma - Chromosomal instability . \\ Copy-number alterations - Array-based comparative \\ genomic hybridization - Metastasis risk
}

\begin{abstract}
Background: To examine whether gastric carcinoma (GC) with chromosomal instability (CIN-type GC), the largest category in the Cancer Genome Atlas classification, consists of a single genetic lineage, we conducted a multisampling analysis of genomic DNA copy-number profile. Methods: We performed array-based comparative genomic hybridization using formalin-fixed paraffin-embedded tissues from 54 gland-forming GCs containing a total of 106 DNA samples from mucosal, extramucosal invasive, and lymph node lesions. Microarray data were analyzed by unsupervised hierarchical clustering and penetrance plots. Epstein-Barr virus infection status and mismatch repair (MMR) enzyme-silencing/p53/mucin expression were examined by in situ hybridization and immunohistochemistry, respectively. Results:
\end{abstract}

The samples examined were divided into gain-rich cluster $A$ and loss-rich cluster $B$, which were different in tumor locus and patient age. The T1/T2-4 ratio, the frequency of small cancers (diameter $\leq 2-4 \mathrm{~cm}$ ), and intestinal mucin expression were higher in cluster B than in cluster $A$, but there were no significant differences in the frequencies of MMR silencing, mutant p53 pattern, and lymph node metastasis between the 2 clusters. Conclusions: We demonstrated that CIN-type GC could be categorized into 2 genetic lineages which are different in terms of rapidity of local extension but similar in terms of nodal metastasis risk.

○ 2019 S. Karger AG, Basel

\section{Introduction}

Gastric carcinoma (GC) is the fifth most common malignant neoplasm and the third leading cause of cancerrelated deaths in both sexes worldwide [1]. Recently, the Cancer Genome Atlas (TCGA) classification of GC included 4 subtypes: Epstein-Barr virus (EBV)-positive
KARGER

() 2019 S. Karger AG, Basel

E-Mail karger@karger.com

www.karger.com/pat
Prof. Hiroyuki Sugihara

Division of Molecular and Diagnostic Pathology, Department of Pathology

Shiga University of Medical Science, Seta-Tsukinowa-cho

Ōtsu, Shiga 520-2192 (Japan)

E-Mail sugihara@belle.shiga-med.ac.jp 
GCs, microsatellite-unstable GCs, genomically stable GCs, and GCs with chromosomal instability (CIN) [2]. It remains unclear how the risk of progression from early to advanced stage, or metastasis risk is assessable and whether the most common CIN-type GCs are genetically homogeneous.

Early GC is defined as a tumor that is limited to the mucosal and submucosal layers, irrespective of the lymph node status. In Japan, early GCs account for approximately $40-60 \%$ of all GC cases [3-5], most of which are detected by endoscopy. Endoscopic resection (ER) of early GCs, which has become the standard treatment in Japan, is gaining greater acceptance worldwide [6]. However, indications for ER of early GC are limited to those with a very low risk of lymph node metastasis; glandforming tumors $\leq 2 \mathrm{~cm}$ at clinical pTla stage [7]. When the endoscopic submucosal dissection (ESD) specimen is pathologically assessed as noncurative, additional treatment follows to prevent recurrence. However, the subsequently removed gastric tissue frequently does not show any evidence of tumor spread or metastasis.

The extent to which early detection and treatment contribute to the reduction of mortality in these patients depends on the lineage continuity between the endoscopically resectable lesions and advanced cancer. In our previous studies, we applied genomic DNA copy-number alteration (CNA) profiling to precursor lesions as well as to early and advanced GC specimens, and classified GC samples using unsupervised hierarchical cluster analysis. Using this approach, we demonstrated that virtually all undifferentiated (diffuse) early GC cases were considered to become advanced [8] whereas around $20 \%$ of noninvasive (gland-forming) neoplasms were considered to eventually become invasive [9]. In this study, we utilized this approach on multiple samples from mucosal, extramucosal invasive, and metastatic lymph node lesions of individual tumor specimens to confirm the consistency of individual-specific changes, and examine the changes associated with tumor progression.

\section{Patients and Methods}

\section{Materials}

This study used formalin-fixed paraffin-embedded tissue specimens from 57 invasive gastric adenocarcinomas including 22 intramucosal, 6 submucosal (including 1 collision cancer, counted as 2 distinct tumors), and 29 advanced cancers (including 2 double cancers, each counted as 2 tumors) (online suppl. Table 1; see www.karger.com/doi/10.1159/000494926 for all online suppl. material). Of these tumors, 25 lymph node-positive $(\mathrm{N}+)$ and 22 node-negative (N0) tumors were surgically resected from 44 patients in the period between 1998 and 2014, and 10 mucosal GCs (cases M27 to M36; online suppl. Table 1) were removed by ESD during the 2012-2014 period. For a diagnosis of N0 tumors, $\geq 10$ of the lymph nodes examined had to be free from metastasis [10]. We performed multisampling, including mucosal, invasive, and if present, lymph node metastatic samples in all 27 advanced GC cases included in this study. The third edition of the Japanese Classification of Gastric Carcinoma and pTNM staging were used to determine histological characteristics and tumor stages, respectively [11].

\section{Immunohistochemistry and EBV in situ Hybridization}

Immunohistochemical staining of 4 - $\mu$ m-thick paraffin sections was performed using an automated Ventana Discovery XT system (Tucson, AZ, USA) with heat pretreatment, an amplification kit (Ventana, 760-080) and a DAB detection kit (Ventana, 760-124). The following 2 monoclonal antibodies against 2 mismatch repair (MMR) proteins were used to assess enzyme silencing, which is closely related to microsatellite instability (MSI) [12]: MSH6 (clone 44, Ventana) and PMS2 (clone EPR3947, Cell Marque, Rocklin, CA, USA). A monoclonal antibody to $\mathrm{p} 53$ protein (DO-7, 1:100; Dako, Glostrup, Denmark) was used to assess p53 expression pattern. Mucin phenotype was analyzed immunohistochemically using monoclonal antibodies against MUC2 (clone MRQ-18, Cell Marque), MUC5AC (clone MRQ-19, Cell Marque), MUC6 (clone MRQ-20, Cell Marque) and CD10 (clone 56C6, Dako). The stains were scored according to the percentage of stained neoplastic cells and categorized into gastric $(G)$, intestinal (I), null (N), $\mathrm{G}>\mathrm{I}$, and $\mathrm{I}>\mathrm{G}$ phenotypes based on the previous study [9]. A polyclonal antibody to SEMA3E protein (1:500; Atlas Antibodies, Bromma, Sweden) was used to evaluate the expression of the SEMA3E gene.

Loss of MMR enzyme expression (LOM) was defined by a complete absence of tumor nuclear staining in specimens with a retention of MMR enzyme expression (ROM) in the nuclei of normal glands and lymphocytes (internal control) [12]. The p53 staining pattern was classified as diffuse, regional, sporadic, and null; the first, second and last of these were considered to reflect mutation, and the sporadic pattern was considered a wild-type (WT) pattern.

EBV status was determined by EBV-encoded small RNA (EBER) in situ hybridization [13] using the INFORM EBER Probe (Ventana, 800-2842, Mannheim, Germany).

\section{Genomic DNA Extraction}

Genomic DNA was extracted from 5- $\mu$ m-thick tumor and normal gland (reference) sections using laser microdissection (LMD6000; Leica Microsystems, Wetzlar, Germany). Samples were obtained from an area of $\geq 6 \mathrm{~mm}^{2}$ in which $\geq 90 \%$ of the cells were neoplastic. A proteinase K solution $(200 \mu \mathrm{g} / \mathrm{mL})$ was used for digestion of the dissected tumor and reference samples for $70 \pm$ $2 \mathrm{~h}$ at $37^{\circ} \mathrm{C}$, followed by phenol/chloroform DNA extraction. DNA quality assessment was based on a cut-off A260/A280 ratio of $>1.5$, a cut-off A260/A230 ratio of $>1.0$, and the concentration of double-stranded DNA.

\section{Whole-Genome Amplification}

We used the GenomePlex whole-genome amplification kit (WGA2 Kit; Sigma, St. Louis, MO, USA) for DNA amplification according to the manufacturer's protocol [14]. 


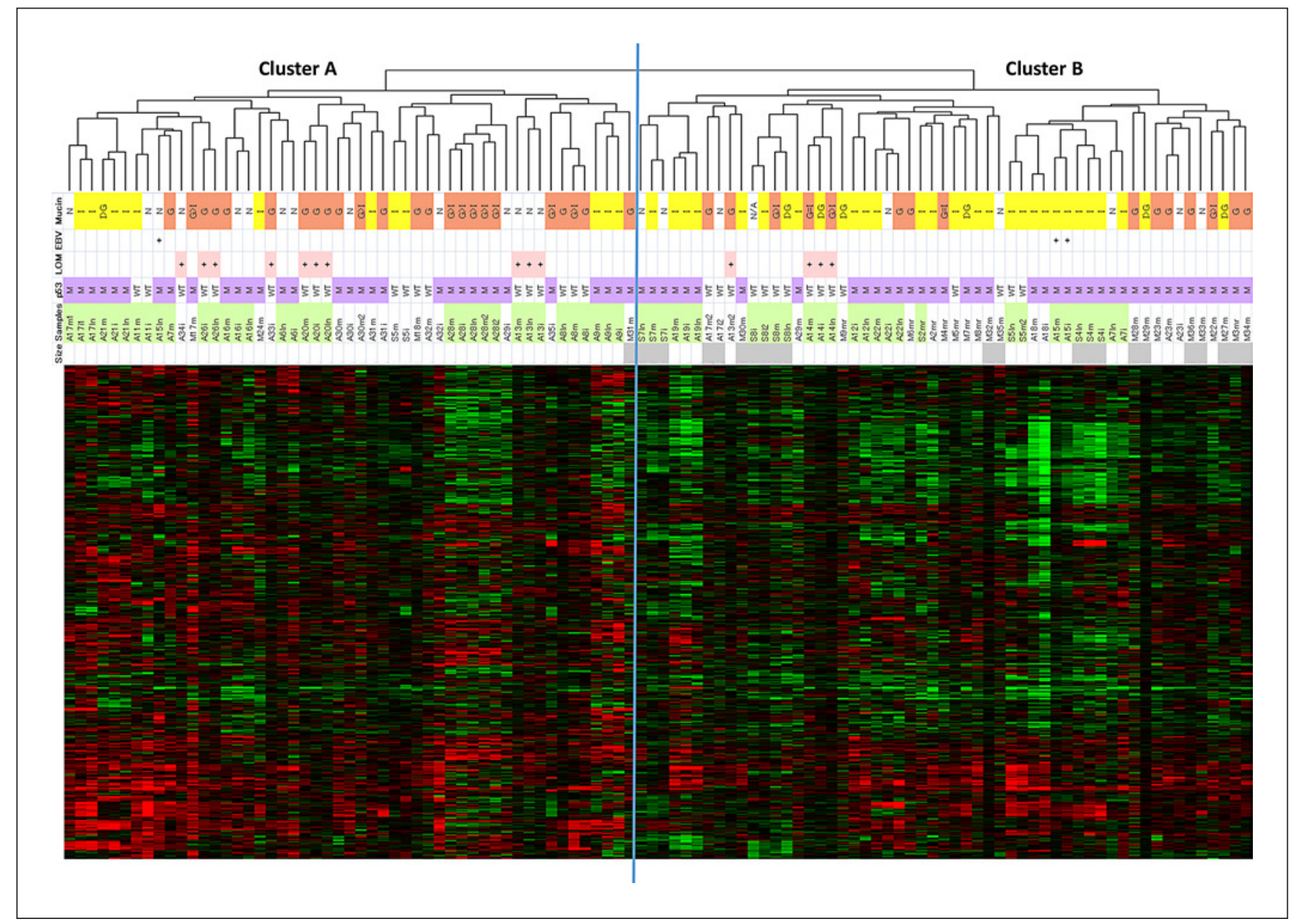

Fig. 1. Unsupervised clustering analysis of 106 samples using the genes/markers of $\geq 5$-probe size. Genomic copy-number gains and losses of genes/markers are indicated by red and green squares, respectively, on the clustering heat map. The clustering results are related to tumor size (gray squares: $\leq 2 \mathrm{~cm}$ ), the presence of lymph node metastasis (marked by a green background of sample names), mutant pattern (M, purple) of p53 immunohistochemistry (WT, wild-type), loss of mismatch repair enzyme expression (LOM, pink), EBV infection status, and mucin phenotype (I/G/N, intestinal/gastric/null). Intestinal-predominant (I and $\mathrm{I}>\mathrm{G}$ ) and gastricpredominant $(\mathrm{G}$ and $\mathrm{G} \geq \mathrm{I})$ phenotypes are marked by a yellow and brown background, respectively.

\section{Array-Based Comparative Genomic Hybridization}

For array-based comparative genomic hybridization (aCGH), 60 -mer length oligonucleotide probes were used according to the manufacturer's guidelines [15]. From each tumor specimen, a set of genomic DNA samples, one from nonneoplastic glands as a reference tumor and the others from tumor parts, were labeled using cyanine 5 and cyanine 3 , respectively, prior to competitive microarray hybridization (SurePrint G3 CGH Microarray 8x60K, GPL10152 62,976 probes). The intensity of all hybridized probes was captured and qualified by a DNA microarray scanner (Feature Extraction software v10.7.3.1), followed by calculation of the ratio of tumor and reference fluorescence intensities. Next, chromosomal patterns within the microarray profiles were visualized, detected, and analyzed by Agilent CGH analytic software using the UCSC Genome Browser according to the latest resource content: hg19 assembly-Design ID 021429 (GRCh Build 37). Definition of genomic copy-number gain and loss, and amplification, were based on $\log _{2}$ of the tumor-to-reference $(T / R)$ ratios which were $>0.3219,<-0.3219$, and $>1$, respectively. The microarray data were registered in the Gene Expression Omnibus (GEO) database (accession No. GSE108507).

\section{Clustering Algorithm}

Before performing the cluster analysis, average $\mathrm{T} / \mathrm{R}$ ratio of the probes within each gene was calculated to intensify the signal-tonoise ratio in hybridization analysis. Given that samples from the same tumor might share random generation of gene CNAs and tissue environmental selection, different parts of a tumor might have similar CNA profiles in the presence of a common carcinogenetic process $[8,9]$. CNA profiles that have a low noise level 


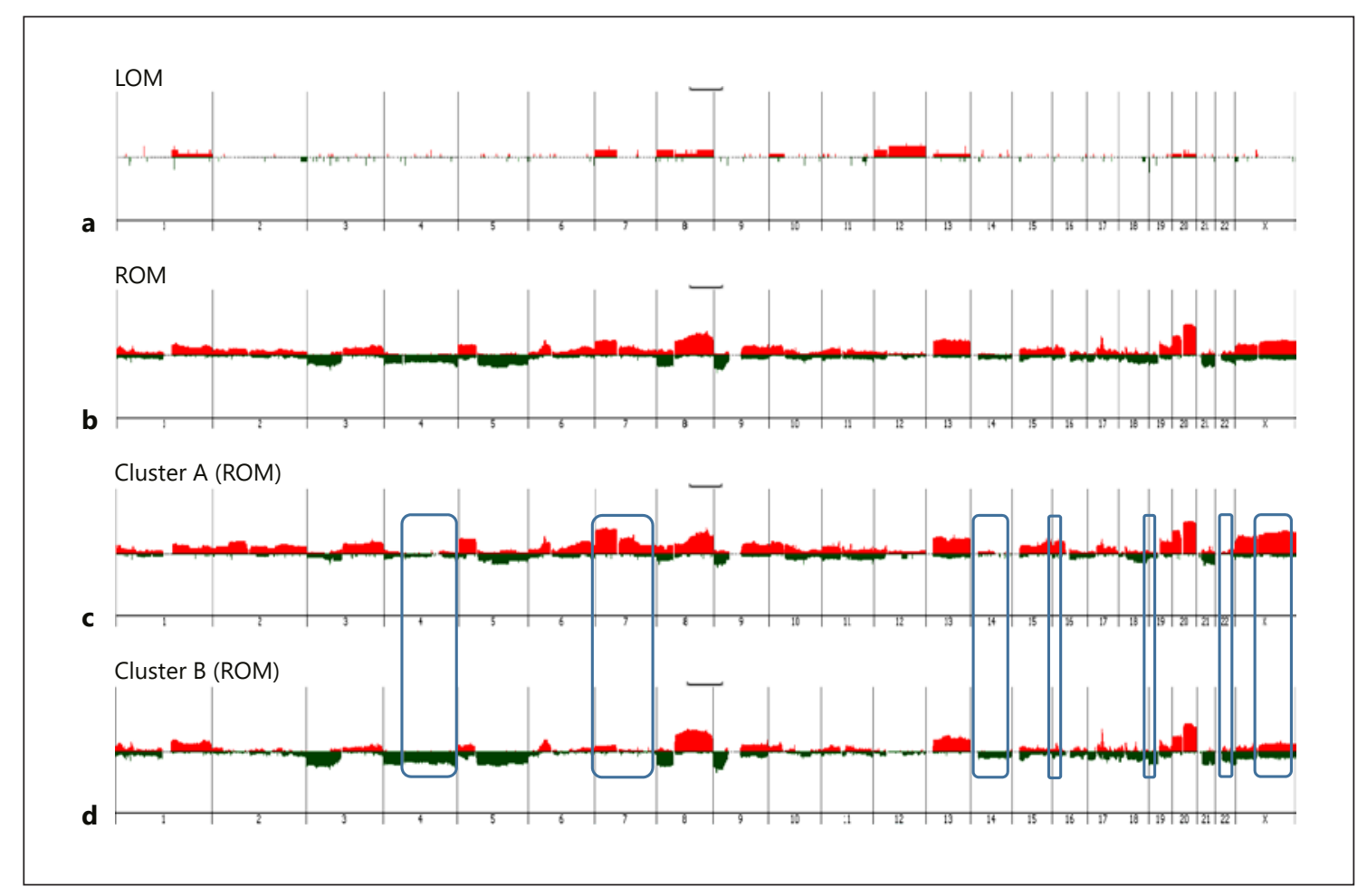

Fig. 2. Frequency of copy-number alterations at the chromosome level (penetrance plots) compared between the tumors with and those without mismatch repair (MMR) enzyme silencing and between clusters A and B. Statistically significant differences are marked with blue frames. a, b LOM, loss of MMR enzyme expression; ROM, retention of MMR enzyme expression. The sample numbers of LOM and ROM were 14 and 92, respectively. c, d Clusters A and B consisted of 41 and 51 samples, respectively.

could satisfy this internal standard. Gene size, which is defined as the number of probes in each gene, impacts the noise-canceling function of the average of $T / R$ ratio whereas clustering reproducibility depends on the number of genes. For smaller gene numbers, the reproducibility of clustering analysis becomes lower, but gene size is larger. Thus, for determining optimal gene size and number, the clustering analysis was repeatedly performed using the Clustering 3.0 (v1.52) software and TreeView (v1.1.6r2) [8,9]. The unsupervised clustering analysis was based on genomic copy-number profile resemblance, and complete linkage and uncentered correlation distance were applied.

Genes Showing Significantly Different CNAs between Clusters

Welch's $t$ test was used between average $\log _{2} \mathrm{~T} / \mathrm{R}$ values of total A cluster and total B cluster samples for 14,977 protein-coding genes. Bonferroni correction was used to correct for multiple comparisons.

\section{Statistical Analysis}

We used Microsoft Office Excel 2013 and BellCurve for Excel (Social Survey Research Information Co., Ltd., Tokyo, Japan). Correlations among variables were examined using Fisher's exact test and Welch's $t$ test. Statistical significance was determined at $p<0.05$.

Loss-Rich and Gain-Rich Lineages in GC

\section{Results}

Unsupervised Clustering Analysis of the CNA Profile

To improve the CNA signal-to-noise ratios, probe T/R ratios within a specified gene were averaged ( $T / R$ ratios of 55,142 probes in 30,471 gene regions). To classify the samples solely by the similarity in their CNA profile, unsupervised cluster analysis was performed. To determine the optimal clustering condition, we repeated the clustering 9 times using varying gene sizes, from 9,487 genes with $\geq 2$ probes to 370 genes with $\geq 10$ probes [9]. We correlated the neighboring or splitting sample distribution in the dendrogram with the presence of large chromosomal changes common to all samples for each case, which we defined as stemline changes. For this purpose, we prepared penetrance plots of individual samples (online suppl. Fig. 1).

Of these 9 conditions, we chose the condition including genes with $\geq 5$ probes because the neighboring sample distribution in the dendrogram was best correlated with the presence of stemline changes. In the chosen condition, out of the 27 cases included in the multisampling, 21 
Table 1. Differences between cluster A and cluster B, mucosal and invasive/metastatic samples, and N0 and N1-3 in the frequency of chromosomal copy-number changes in ROM tumors

\begin{tabular}{|c|c|c|c|c|c|c|c|c|c|}
\hline & \multicolumn{2}{|c|}{ Samples, $n$} & $p$ value $^{\mathrm{a}}$ & \multicolumn{2}{|c|}{ Samples, $n$} & $p$ value $^{\mathrm{b}}$ & \multicolumn{2}{|c|}{ Samples, $n$} & $p$ value $^{\mathrm{c}}$ \\
\hline $3 p-$ & 7 & 14 & 0.3192 & 6 & 15 & 0.0028 & 17 & 4 & 0.0114 \\
\hline $3 q+$ & 10 & 6 & 0.1660 & 6 & 10 & 0.0967 & 11 & 5 & 0.2792 \\
\hline $4 \mathrm{p}-$ & 4 & 12 & 0.1020 & 5 & 11 & 0.0260 & 14 & 2 & 0.0097 \\
\hline $5 q-$ & 8 & 16 & 0.2374 & 11 & 13 & 0.2306 & 19 & 5 & 0.0083 \\
\hline $7 \mathrm{p}+$ & 23 & 7 & $<0.0001$ & 9 & 21 & 0.0003 & 24 & 6 & 0.0015 \\
\hline $7 q+$ & 12 & 2 & 0.001 & 3 & 11 & 0.0064 & 13 & 1 & 0.0025 \\
\hline $8 \mathrm{p}-$ & 6 & 16 & 0.0853 & 7 & 15 & 0.0067 & 19 & 3 & 0.0011 \\
\hline $8 \mathrm{q}+$ & 19 & 23 & 1.0000 & 23 & 19 & 0.6746 & 25 & 17 & 0.5309 \\
\hline $9 \mathrm{p}-$ & 7 & 17 & 0.0969 & 9 & 15 & 0.0300 & 18 & 6 & 0.0320 \\
\hline $16 \mathrm{p}+$ & 11 & 3 & 0.0077 & 6 & 8 & 0.2525 & 11 & 3 & 0.0806 \\
\hline $17 \mathrm{p}-$ & 2 & 10 & 0.0592 & 6 & 6 & 0.7554 & 9 & 3 & 0.2144 \\
\hline $18 \mathrm{q}-$ & 7 & 10 & 0.7938 & 6 & 11 & 0.0565 & 12 & 5 & 0.1877 \\
\hline $19 \mathrm{p}-$ & 2 & 12 & 0.0181 & 5 & 9 & 0.0851 & 10 & 4 & 0.2486 \\
\hline $19 q-$ & 1 & 4 & 0.3764 & 1 & 4 & 0.1587 & 2 & 3 & 0.6528 \\
\hline $20 \mathrm{q}+$ & 27 & 29 & 0.3996 & 28 & 28 & 0.0847 & 39 & 17 & 0.0011 \\
\hline $22 \mathrm{q}-$ & 0 & 11 & 0.0009 & 4 & 7 & 0.1934 & 8 & 3 & 0.3343 \\
\hline $\mathrm{Xp}+$ & 18 & 5 & 0.0002 & 8 & 15 & 0.0147 & 19 & 4 & 0.0032 \\
\hline $\mathrm{Xq}-$ & 1 & 5 & 0.2202 & 1 & 5 & 0.0796 & 6 & 0 & 0.0316 \\
\hline $\mathrm{Xq}+$ & 19 & 8 & 0.0024 & 10 & 17 & 0.0121 & 20 & 7 & 0.0231 \\
\hline
\end{tabular}

ROM, retention of mismatch repair enzyme expression; $M$, mucosal; I, invasive; LNM, lymph node metastasis; N0, no evidence of nodal involvement; N1-3, nodal tumor involvement grades $1-3$. $p$ values $<0.05$ appear in bold type.

${ }^{\mathrm{a}}$ Cluster A vs. cluster B; ${ }^{\mathrm{b}} \mathrm{M}$ vs. I + LN; ${ }^{\mathrm{c}} \mathrm{N} 1-3$ vs. N0.

cases showed a neighboring pattern and 6 showed a splitting pattern in the sample distribution (Fig. 1). Of the 21 neighboring pattern cases, stemline chromosomal changes were detected in 17 cases with ROM; in 4 cases with LOM, no changes were detected (online suppl. Fig. 1). The 6 splitting pattern cases included 2 double cancers (case\# A13 and A17), 3 cancers with almost no common chromosomal changes in the samples (case\# S5, A15, and A29), and 1 cancer with stemline changes but splitting between the samples with and without 1p/q+ (case\# A7).

\section{Clustering Results and Their Relationship to MMR \\ Enzyme and p53 Expression, EBV Infection, and \\ Mucin Phenotype}

Consequently, a total of 106 samples were classified into 2 major clusters, A and B (Fig. 1). The heat map indicated that there was a copy-number gain area common to both clusters. Clusters A and B were characterized by the presence of gain-rich and loss-rich areas, respectively. LOM samples characteristically showed dark on the heat map. There were no differences in the frequencies of LOM and mutant $\mathrm{p} 53$ pattern between the 2 clusters. The LOM samples were not distributed in the clusters. Additionally, we confirmed that MMR enzyme-silencing and mutant p53 pattern were mutually exclusive (Fig. 1). The tumors we examined involved only 1 case with EBV infection. The staining results of mucin phenotype are shown in online supplementary Table 1 . We found that the relative frequency of gastric-predominant phenotype ( $\mathrm{G}$ and $\mathrm{G} \geq \mathrm{I}$ )/ null type $(\mathrm{N})$ was significantly lower in invasive/metastatic parts than in mucosal parts, whereas intestinal-predominant phenotype ( $\mathrm{I}$ and $\mathrm{I}>\mathrm{G}$ ) was almost constant between these parts (online suppl. Fig. 2). Therefore, we can tentatively regard the intestinal expression as a progression- 


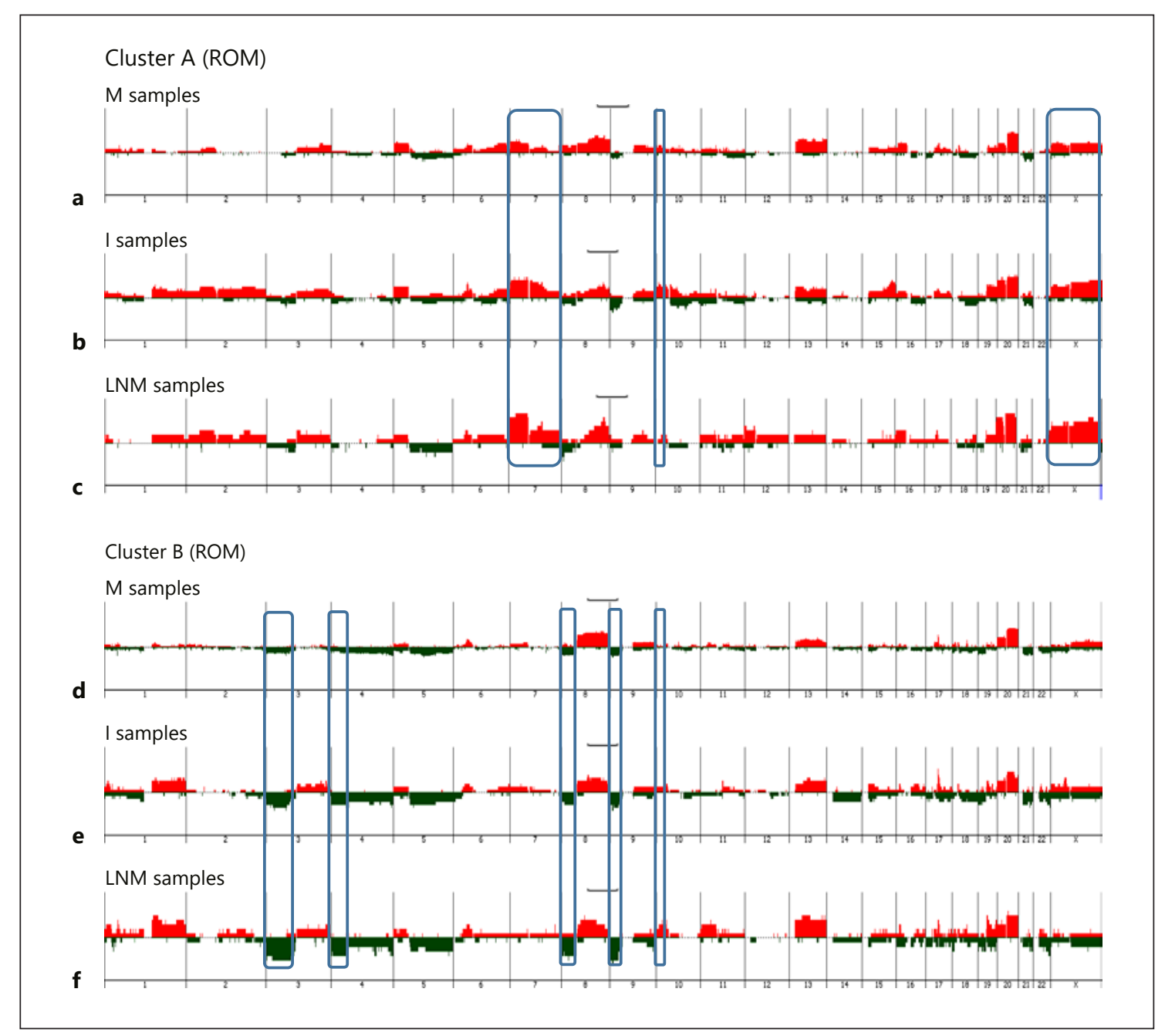

Fig. 3. Penetrance plots of ROM tumors in clusters A and B. Cluster-specific, progression-related significant changes are marked with blue frames. a, d Mucosal (M) samples. b, e Extramucosal invasive (I) samples. c, f Lymph node metastasis (LNM) samples. a-f Sample numbers were 18, 15, 8, 31, 12, and 8, respectively.

independent lineage marker. This marker expression was more common in cluster B than in cluster A.

\section{Chromosomal CNAs}

The penetrance plots of the tumors with LOM demonstrated infrequent gains (such as $12 \mathrm{q}+$, which was rare in the ROM samples) and scarce losses, whereas gains and losses were conspicuous in the ROM tumors (Fig. 2a, b), which were divided into 2 patterns by clustering (Fig. 2c, d). The changes common to both clusters were $8 \mathrm{q}+, 13 \mathrm{q}+$, $20 \mathrm{q}+$, and $5 \mathrm{q}-$. Clusters $A$ and $B$ were characterized by gain-rich $(7 \mathrm{p} / \mathrm{q}+, \mathrm{Xp} / \mathrm{q}+$, etc.) and loss-rich $(4 \mathrm{p} / \mathrm{q}-$, etc.), respectively (Fig. 2c, d). Statistical analyses are shown in Table 1. The mucosal, invasive, and metastatic ROM sam- ples were compared in clusters $\mathrm{A}$ and $\mathrm{B}$ (Fig. 3). The changes common to clusters $A$ and $B(8 q+, 13 q+, 20 q+$, and $5 \mathrm{q}-)$ were already present in the mucosal samples, whereas cluster-specific changes (such as $4 \mathrm{p} / \mathrm{q}-$ and $7 \mathrm{p} /$ $\mathrm{q}+$ ) accumulated during the progression to invasion and metastasis. These were confirmed by statistical analysis of the frequency of each chromosomal CNA, which were determined by counting using the penetrance plot of each sample (online suppl. Fig. 1) and compared between the mucosal and extramucosal (invasive and metastatic) samples (Table 1). In contrast, no such differences were detected between the invasive and metastatic samples. Table 1 also shows the metastasis-related chromosomal changes. The chromosomal changes common to clusters 
Fig. 4. Evolutionary tree of gland-forming gastric carcinoma reconstructed from $\mathrm{Ta}-$ ble 1 and Fig. 2, 3. LOM/ROM, loss/retention of mismatch repair enzyme expression. Chromosomal gains and losses are shown as red and green characters. Bold/ normal letters indicate frequent/infrequent significant changes. Chromosomal parts in parentheses indicate insignificant but characteristic changes.

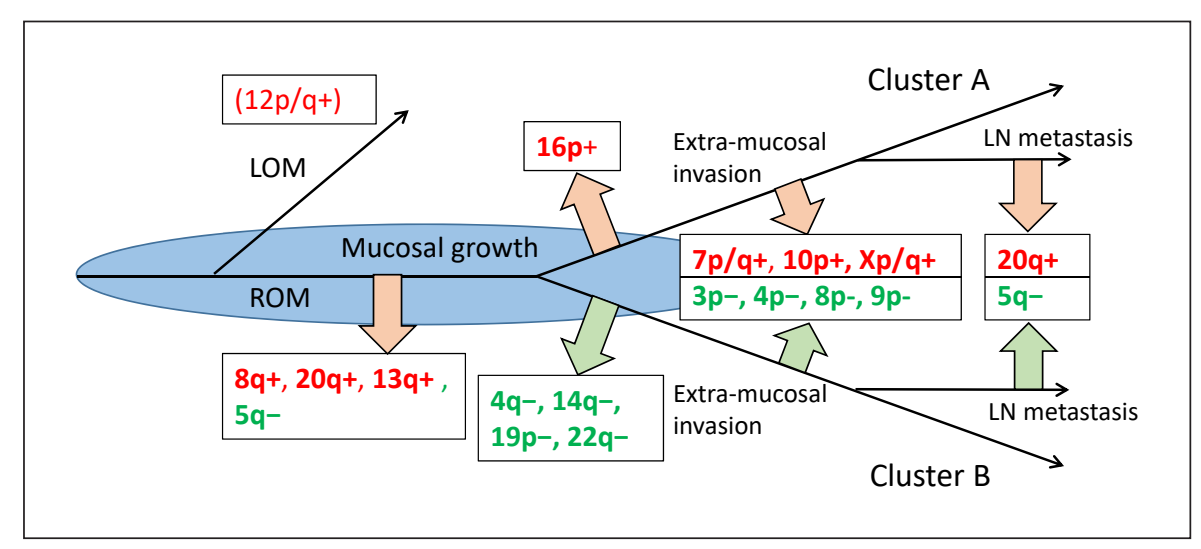

$A$ and $B$ were not related or were weakly related to metastasis, but the cluster-specific changes, such as $7 \mathrm{p} / \mathrm{q}+$ in cluster $A$ and $3 p-, 4 p-, 5 q-$, and $8 p-$ in cluster $B$, were significantly related to lymph node metastasis. The chromosomal changes detected are summarized as an evolutionary tree in Figure 4.

\section{Clinicopathological and Molecular Characteristics of} the Clusters

Clinicopathological characteristics and molecular changes were compared between the tumors of clusters A and $\mathrm{B}$ (Table 2). The tumor locus and patient age were different between clusters A and B. In this tumor-based analysis, representative phenotype in each submucosal or advanced cancers was defined to that of the invasive sample, and we could also confirm that the intestinal expression was more frequent in cluster B than in cluster $\mathrm{A}$.

The T1/T2-4 ratio and the frequency of small cancers (diameter $\leq 2-4 \mathrm{~cm}$ ) were higher in cluster $\mathrm{B}$ than in cluster A, suggesting that GCs in cluster A grew more rapidly than those in cluster B. Nine of the 10 ESD specimens examined were classified into cluster B. There were, however, no significant differences in the frequencies of lymph node metastasis.

\section{Genes Showing Significantly Different CNAs in the Clusters}

We identified 32 genes that significantly contributed to the difference in clusters A and B. Of these, 12 were related to growth, as shown in online supplementary Figure 2. When the gene function and the direction of CNAs were consistent, we regarded the CNAs as putative driver, and when inconsistent, as putative passenger. There were 5 putative driver tumor suppressor genes (CACNA2D3, $P T P R G$, and LRIG1 at chromosome 3p, SLIT2 at chromosome 4p, and FSTL5 at chromosome 4q) and 1 possible driver protooncogene (semaphorin $3 \mathrm{E}[S E M A 3 E]$ ) at $7 \mathrm{q}$. However, immunohistochemically, there was no difference in the expression level of SEMA3E protein between 10 tumors that showed the greatest copy-number gains and those with the greatest copy-number losses.

To compare the growth activity between cluster $\mathrm{A}$ and cluster B tumors, we examined the frequency of amplification of growth-related genes, which is a characteristic of CIN-type GC. Of the 55 receptor tyrosine kinases (RTKs) examined, copy-number gains of 12 RTKs, including EGFR, ERBB3, FGFR4, and EPHB3, and amplification of LMTK2, EPHB3, EPHB4 showed significantly different frequencies in cluster A and cluster B. All of them were detected more frequently in cluster $A$ than in cluster B (online suppl. Table 2).

\section{Discussion}

Using the multisampling method, we could demonstrate not only the differences among samples but also the reproducibility of changes common to all samples in individual GC patients. To assess the reproducibility of this approach as well as classify samples, we applied unsupervised, hierarchical clustering analysis to the multisampling aCGH data of 57 early and advanced gland-forming GC specimens. We selected the clustering condition that showed the highest agreement between the neighboring pattern in the clustering dendrogram and the presence of stemline changes. Even in the absence of stemline changes among the samples from individual tumors such as the LOM tumors, the mucosal and invasive/metastatic samples often showed a neighboring position in the dendrogram, likely reflecting a similarity among gene-level CNAs. CNA profiles are considered as individual, tumorspecific, and progression-independent lineage markers. 
Table 2. Differences between clusters A and B regarding clinicopathological factors in 57 tumors

\begin{tabular}{|c|c|c|c|c|}
\hline & Cluster A & Cluster B & Total & $p$ value \\
\hline \multicolumn{5}{|l|}{ Gender $^{\mathrm{a}}$} \\
\hline Male & 12 & 22 & 34 & \multirow[t]{2}{*}{0.5451} \\
\hline Female & 8 & 9 & 17 & \\
\hline $\operatorname{Age}^{\mathrm{a}}$, years $($ mean $\pm S D)$ & $75.35 \pm 8.65$ & $68.55 \pm 7.24$ & & 0.006 \\
\hline \multicolumn{5}{|l|}{ Tumor locus } \\
\hline Upper (U) & 3 & 9 & 12 & \multirow{3}{*}{$\begin{array}{l}\mathbf{0 . 0 0 3 8} \\
(\mathrm{U}+\mathrm{M} \text { vs. } \mathrm{L})\end{array}$} \\
\hline Middle (M) & 7 & 19 & 26 & \\
\hline Lower (L) & 13 & 6 & 19 & \\
\hline \multicolumn{5}{|l|}{ Tumor size } \\
\hline$\leq 2 \mathrm{~cm}$ & 1 & 14 & 15 & \multirow[t]{2}{*}{0.0019} \\
\hline$>2 \mathrm{~cm}$ & 22 & 20 & 42 & \\
\hline$\leq 3 \mathrm{~cm}$ & 5 & 19 & 24 & \multirow[t]{2}{*}{0.0143} \\
\hline$>3 \mathrm{~cm}$ & 18 & 15 & 33 & \\
\hline$\leq 4 \mathrm{~cm}$ & 9 & 26 & 35 & \multirow[t]{2}{*}{0.0062} \\
\hline$>4 \mathrm{~cm}$ & 14 & 8 & 22 & \\
\hline$\leq 5 \mathrm{~cm}$ & 14 & 27 & 41 & \multirow[t]{2}{*}{0.1454} \\
\hline$>5 \mathrm{~cm}$ & 9 & 7 & 16 & \\
\hline \multicolumn{5}{|l|}{ Local extension } \\
\hline $\mathrm{T} 1$ & 5 & 23 & 28 & \multirow[t]{2}{*}{0.0011} \\
\hline $\mathrm{T} 2-4$ & 18 & 11 & 29 & \\
\hline \multicolumn{5}{|l|}{ Grade of nodal tumor involvement } \\
\hline No & 12 & 23 & 35 & \multirow[t]{2}{*}{0.2769} \\
\hline N1-3 & 11 & 11 & 22 & \\
\hline \multicolumn{5}{|l|}{ MMR enzyme expression } \\
\hline LOM & 5 & 2 & 7 & \multirow[t]{2}{*}{0.1057} \\
\hline ROM & 18 & 32 & 50 & \\
\hline \multicolumn{5}{|l|}{ EBV infection } \\
\hline Positive & 0 & 1 & 1 & \multirow[t]{2}{*}{1.0000} \\
\hline Negative & 23 & 33 & 56 & \\
\hline \multicolumn{5}{|l|}{ p53 Expression } \\
\hline Diffuse/regional/null & 13 & 26 & 39 & \multirow[t]{2}{*}{0.1497} \\
\hline Sporadic & 10 & 8 & 18 & \\
\hline \multicolumn{5}{|l|}{ Phenotype } \\
\hline Intestinal-predominant & 5 & 17 & 23 & \multirow[t]{2}{*}{0.0264} \\
\hline Nonintestinal-predominant & 17 & 15 & 32 & \\
\hline
\end{tabular}

During the development of individual tumors, random changes as well as essential genetic and epigenetic changes accumulate in a time-dependent manner based on genetic instability, and these changes undergo natural selection by the tissue environment. In this evolutionary process, genomic alteration profiles might become unique to each individual tumor and might converge in several types by natural selection [16]. We attempted to reveal such converged genotypes using unsupervised hierarchical clustering.

The tumors included in our analyses were mostly of the CIN-type and partly of the MSI-type in the TCGA classification, but EBV-related GCs were infrequent. A cancer with LOM, which largely overlaps with the MSI phenotype, is characterized by a low frequency of chromosomal changes and the presence of the unique $12 \mathrm{q}+$ (Fig. 2a, b), and it may have branched off from the main trunk of gland-forming GCs at an earlier stage of GC development (Fig. 4). Accordingly, it has been reported that GCs of MSI phenotype show a frequency of chromosomal CNAs lower than in GCs without MSI and are associated with a high DNA methylation status, even at intramucosal stages [17]. 
We classified ROM GC samples (GCs excluding LOM) into 2 clusters: A (ROM) and B (ROM) (Fig. 2c, d). Based on the resemblance of gene-level CNA profiles, our clustering approach successfully disclosed 2 distinct genetic lineages (gain-rich and loss-rich) that have not been clearly recognized thus far. In this study, we analyzed the sequential accumulation of chromosomal CNAs during progression from mucosal to invasive/metastatic growth in each lineage (Fig. 3). Both lineages could have derived from a common trunk with $8 \mathrm{q}+, 13 \mathrm{q}+, 20 \mathrm{p} / \mathrm{q}+$, and $5 \mathrm{q}-$, commonly detected in both cluster A and cluster B. These findings partly confirm the report of Uchida et al. [18], who reported $8 \mathrm{p} \pm$ and $5 \mathrm{q}-$ as early changes of $\mathrm{GC}$, and then cluster-specific later changes, such as $7 \mathrm{pq}+$ in cluster $\mathrm{A}$ and $4 \mathrm{pq}-$ in cluster B (Fig. 4).

Next, we classified all samples into 2 clusters, A and B. There appeared to be little association between this cluster classification and TCGA GC subtypes (Table 2). The tumor locus and age of patients were different in cluster $\mathrm{A}$ and cluster $\mathrm{B}$. The age difference might be explained by the difference in the frequency of early GCs, whereas the locus difference suggests that this clustering might reflect distinct genetic lineages. This was also supported by mucin phenotyping; progression-independent intestinal expression was significantly different in clusters A and B. Comparison of other clinicopathological factors between clusters A and B indicated that the tumors in cluster A grew more rapidly (larger, deeper tumors) than those in cluster B. ESD specimens were mostly included in the latter. These findings implied that early detection and treatment were more difficult for cluster A than cluster B tumors (Fig. 5).

Rapid growth in the gain-rich lineage (cluster A) might be partly due to the copy-number gains/amplifications of the RTKs mentioned above and the growth-related genes in chromosome 7, including RalA [19], EGFR [20], MAFK [21], GLI3 [22], CUL1 [23], and SEMA3E [24, 25] (online suppl. Table 2). Among these, only SEMA3E exhibited significant copy-number differences after Bonferroni correction between clusters A and B. However, its expression is reportedly enhanced [24] or suppressed [25] in GCs. Our immunohistochemical studies for SEMA3E failed to demonstrate a significant association between protein expression level and gene copy number, and we cannot regard this gene as a functional driver or suppressor. Rather, detection of immunoreactivity for amplified growth-related genes may be more promising as cluster A markers. In the loss-rich lineage (cluster B), the abovementioned tumor suppressor genes on $3 \mathrm{p}$ and $4 \mathrm{p} / \mathrm{q}$ showed significant differences between clusters A and B.

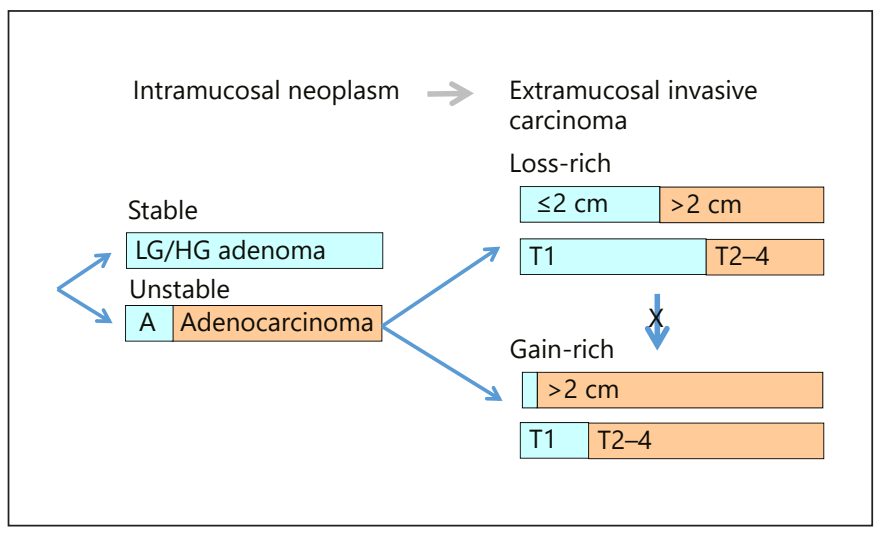

Fig. 5. Progression model of a gland-forming gastric neoplasm from an intramucosal neoplasm to invasive carcinoma.

The silencing of CACNA2D3 [26] and PTPRG [27], in particular, was reported to play a role in gastric tumorigenesis.

There were no significant differences in the risk of lymph node metastasis between the rapidly and slowly growing lineages, but there were lineage-specific, invasion/metastasis-related chromosomal changes. These findings suggest that metastasis risk is determined not at an earlier but a later stage during tumor development (Fig. 4).

These conclusions have several clinical implications for early detection and treatment strategies. This study was conducted with a null hypothesis that there are indolent cancers treated as cancers, which are associated with very low mortality risk. We previously demonstrated that high-grade and low-grade gastric adenomas, the former treated as carcinoma in Japan, are such tumors. Only around $20 \%$ of these tumors were inferred to progress to overt GC [9] (Fig. 5). This study shows that there was no inherently indolent CIN-type early GC cluster and that the 2 lineages had an equal potential for metastasis. Our findings also demonstrated that GCs detected at an early stage were biased to a slow-growing loss-rich lineage. The prevalent use of early detection by endoscopy and the subsequent treatment contribute to a reduction in the incidence of advanced GC and age-adjusted mortality rates; however, these reductions have been smaller than expected compared with the reduction in GC morbidity [28]. One major cause of this might be the increase in the percentage of older patients. Another factor could be the failure of early detection of GCs with a rapidly growing gainrich lineage, which remains a challenge that should be addressed in future studies. 


\section{Acknowledgements}

The authors thank Associate Professor Suzuko Moritani and Professor Ryoji Kushima, Department of Pathology, Shiga University of Medical Science Hospital, for their kind support for the EBV in situ hybridization.

This study was supported in part by JSPS KAKENHI, grant Nos. JP25460454 and JP16K08689.

\section{Statement of Ethics}

All procedures followed were in accordance with the ethics standards of the responsible committee on human experimentation (institutional and national) and with the Helsinki Declaration of 1964 and later versions. The Institutional Review Board on Medical Ethics at the Shiga University of Medical Science granted permission for conducting this study (permission No. 30-021). A substitute of written informed consent was obtained from all patients included in the study.

\section{Disclosure Statement}

The authors declare that they have no conflicts of interest.

\section{References}

1 Ferlay J, Soerjomataram I, Ervik M, Dikshit R, Eser S, Mathers CR, et al. GLOBOCAN 2012 v1.0, Cancer Incidence and Mortality Worldwide: IARC Cancer Base No. 11 [Internet] Lyon, France: International Agency for Research on Cancer; 2013. [cited 201621 Nov], Available from http://globocan.iarc.fr

2 Bass AJ, Thorsson V, Shmulevich I, Reynolds SM, Miller M, Bernard B, et al.; Cancer Genome Atlas Research Network. Comprehensive molecular characterization of gastric adenocarcinoma. Nature. 2014 Sep;513(7517): 202-9.

3 Everett SM, Axon AT. Early gastric cancer in Europe. Gut. 1997 Aug;41(2):142-50.

4 Shimizu S, Tada M, Kawai K. Early gastric cancer: its surveillance and natural course. Endoscopy. 1995 Jan;27(1):27-31.

5 Tanaka M, Ono H, Hasuike N, Takizawa K. Endoscopic submucosal dissection of early gastric cancer. Digestion. 2008;77(1 Suppl 1): 23-8.

6 Gotoda T. Endoscopic resection of early gastric cancer. Gastric Cancer. 2007;10(1):1-11.

7 Japanese Gastric Cancer Association. Japanese gastric cancer treatment guidelines 2010 (ver. 3). Gastric Cancer. 2011 Jun;14(2):113-23.

8 Sonoda A, Mukaisho K, Nakayama T, Diem VT, Hattori T, Andoh A, et al. Genetic lineages of undifferentiated-type gastric carcinomas analysed by unsupervised clustering of genomic DNA microarray data. BMC Med Genomics. 2013 Jul;6(1):25.

9 Vo DT, Nakayama T, Yamamoto $\mathrm{H}, \mathrm{Mu}-$ kaisho K, Hattori T, Sugihara H. Progression risk assessments of individual non-invasive gastric neoplasms by genomic copy-number profile and mucin phenotype. BMC Med Genomics. $2015 \mathrm{Feb} ; 8(1): 6$.

10 Borie F, Plaisant N, Millat B, Hay JM, Fagniez PL; French Associations for Surgical Research. Appropriate gastric resection with lymph node dissection for early gastric cancer. Ann Surg Oncol. 2004 May;11(5):512-7.

11 Japanese Gastric Cancer Association. Japanese classification of gastric carcinoma: 3rd
English edition. Gastric Cancer. 2011 Jun; 14(2):101-12

12 Mojtahed A, Schrijver I, Ford JM, Longacre TA, Pai RK. A two-antibody mismatch repair protein immunohistochemistry screening approach for colorectal carcinomas, skin sebaceous tumors, and gynecologic tract carcinomas. Mod Pathol. 2011 Jul;24(7):1004-14

13 Okamoto A, Yanada M, Miura H, Inaguma Y, Tokuda M, Morishima S, et al. Prognostic significance of Epstein-Barr virus DNA detection in pretreatment serum in diffuse large Bcell lymphoma. Cancer Sci. 2015 Nov;106(11): 1576-81.

14 Little SE, Vuononvirta R, Reis-Filho IS, Natrajan R, Iravani M, Fenwick K, et al. Array $\mathrm{CGH}$ using whole genome amplification of fresh-frozen and formalin-fixed, paraffinembedded tumor DNA. Genomics. 2006 Feb; 87(2):298-306.

15 Barrett MT, Scheffer A, Ben-Dor A, Sampas N, Lipson D, Kincaid R, et al. Comparative genomic hybridization using oligonucleotide microarrays and total genomic DNA. Proc Natl Acad Sci USA. 2004 Dec;101(51):1776570.

16 Gleaves M. Cancer: The Evolutionary Legacy. Oxford University Press; 2001.

17 Sugai T, Eizuka M, Arakawa N, Osakabe M, Habano W, Fujita Y, et al. Molecular profiling and comprehensive genome-wide analysis of somatic copy number alterations in gastric intramucosal neoplasias based on microsatellite status. Gastric Cancer. 2018 Sep;21(5):76575.

18 Uchida M, Tsukamoto Y, Uchida T, Ishikawa Y, Nagai T, Hijiya N, et al. Genomic profiling of gastric carcinoma in situ and adenomas by array-based comparative genomic hybridization. J Pathol. 2010 May;221(1):96-105.

19 Martin TD, Samuel JC, Routh ED, Der CJ, Yeh JJ. Activation and involvement of Ral GTPases in colorectal cancer. Cancer Res. 2011 Jan;71(1):206-15.

20 Oh HS, Eom DW, Kang GH, Ahn YC, Lee SJ, $\mathrm{Kim} \mathrm{JH}$, et al. Prognostic implications of
EGFR and HER-2 alteration assessed by immunohistochemistry and silver in situ hybridization in gastric cancer patients following curative resection. Gastric Cancer. 2014; 17(3):402-11.

21 Okita Y, Kimura M, Xie R, Chen C, Shen LT, Kojima Y, et al. The transcription factor MAFK induces EMT and malignant progression of triple-negative breast cancer cells through its target GPNMB. Sci Signal. 2017 Apr;10(474):474.

22 Iwasaki $\mathrm{H}$, Nakano K, Shinkai K, Kunisawa $\mathrm{Y}$, Hirahashi M, Oda Y, et al. Hedgehog Gli3 activator signal augments tumorigenicity of colorectal cancer via upregulation of adherence-related genes. Cancer Sci. 2013 Mar; 104(3):328-36.

23 Bai J, Zhou Y, Chen G, Zeng J, Ding J, Tan Y, et al. Overexpression of Cullin1 is associated with poor prognosis of patients with gastric cancer. Hum Pathol. 2011 Mar;42(3):375-83.

24 Maejima R, Tamai K, Shiroki T, Yokoyama M, Shibuya R, Nakamura M, et al. Enhanced expression of semaphorin $3 \mathrm{E}$ is involved in the gastric cancer development. Int J Oncol. 2016 Sep;49(3):887-94.

25 Chen H, Xie GH, Wang WW, Yuan XL, Xing WM, Liu HJ, et al. Epigenetically downregulated Semaphorin 3E contributes to gastric cancer. Oncotarget. 2015 Aug;6(24):20449_ 65.

26 Wanajo A, Sasaki A, Nagasaki H, Shimada S, Otsubo T, Owaki S, et al. Methylation of the calcium channel-related gene, CACNA2D3, is frequent and a poor prognostic factor in gastric cancer. Gastroenterology. 2008 Aug. 135(2):580-90

27 Wu CW, Kao HL, Li AF, Chi CW, Lin WC. Protein tyrosine-phosphatase expression profiling in gastric cancer tissues. Cancer Lett. 2006 Oct;242(1):95-103.

28 Tani MS, Nakanish Y, Ochiai A, Taniguchi H, Sasako M, et al. Chronological trends of gastric carcinoma in Japanese from the pathological view point. Stomach and Intestine (Tokyo). 2005;40:27-36. 